\title{
REMOTE METHOD OF TEMPERATURE MEASUREMENT IN THE FOCUS OF HIGH-TEMPERATURE SOLAR FURNACES
}

\author{
Sirozhiddin Makhmudov ${ }^{1}$, Yuldash Sobirov ${ }^{2,1}$ Abdujabbor Abdurakhmanov $^{2}$ \\ ${ }^{1}$ Tashkent State Technical University named after Islam Karimov, University-2, Tashkent, 100095, Uzbekistan \\ ${ }^{2}$ Material Sciences Institute of Scientific Production Association "Physics-Sun”, Ch. Aytmatov -2b, Tashkent, 100080, Uzbekistan
}

\begin{abstract}
The paper presents a remote method for measuring heated bodies up to $3000{ }^{\circ} \mathrm{C}$ under a concentrated flow of radiant energy from the sun. The article discusses the issue of improving the accuracy of temperature measurement by refining the emissivity of materials during irradiation with a highly concentrated radiant flux.
\end{abstract}

\section{Introduction}

Various thermometers, thermocouples, pyrometers are often used to measure the temperature of heated bodies in high-temperature technological installations [1]. However, measuring the temperature of the body exposed to a highly concentrated flux of solar radiation is a very difficult task. Especially such solar installations as the Big Solar Furnace (BSF) of megawatt capacity of the Institute of Materials Science SPA "Physics-Sun" of the Academy of Sciences of the Republic of Uzbekistan [2]. Stretch the diameter of the focal spot up to $1.5 \mathrm{~m}$, and the body temperature reaches $3000{ }^{\circ} \mathrm{C}$. In the focal zone of BSF, the following high-temperature composite materials are synthesized: $3 \mathrm{Al}_{2} \mathrm{O}_{3} \cdot 2 \mathrm{SiO}_{2} ; \mathrm{Al}_{2} \mathrm{MgO}_{4}$ for the optical industry; $\mathrm{Al}_{2} \mathrm{TiO}_{5} ; \mathrm{CaZrO}_{3}$ - special ceramics with a low coefficient of thermal expansion; $\mathrm{AlGdO}_{3} ; \mathrm{LiAlO}_{2}$ - materials for nuclear reactors; $\mathrm{Li}_{2} \mathrm{O}$ $\mathrm{Al}_{2} \mathrm{O}_{3} \cdot \mathrm{SiO}_{2} \cdot\left(\mathrm{ZrO}_{2}, \mathrm{HfO}_{2}\right)$ - glass-crystalline materials with a low coefficient of thermal expansion and high transparency; $\mathrm{YBa}_{2} \mathrm{Cu}_{3} \mathrm{O}_{7}-\mathrm{X} ; \mathrm{Bi}_{2} \mathrm{Sr}_{2} \mathrm{Ca}_{2} \mathrm{Cu}_{3} \mathrm{Oy}$ - hightemperature superconductors.

In such conditions, the use of thermometers, thermocouples or other measuring probes to measure the local temperatures of the irradiated body is very difficult, not possible in pairs. In one case, the thermometers themselves melt, and in the other case, they show incorrectly in their entirety being in the irradiated area of high radiant flux density.

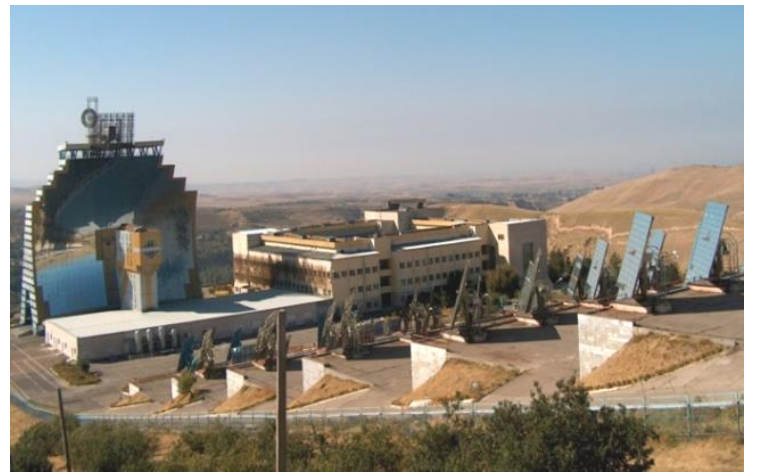

Fig. 1. General view of the Big Solar Furnace of Uzbekistan with a thermal capacity of $1000 \mathrm{~kW}$ in Parkent (Uzbekistan).

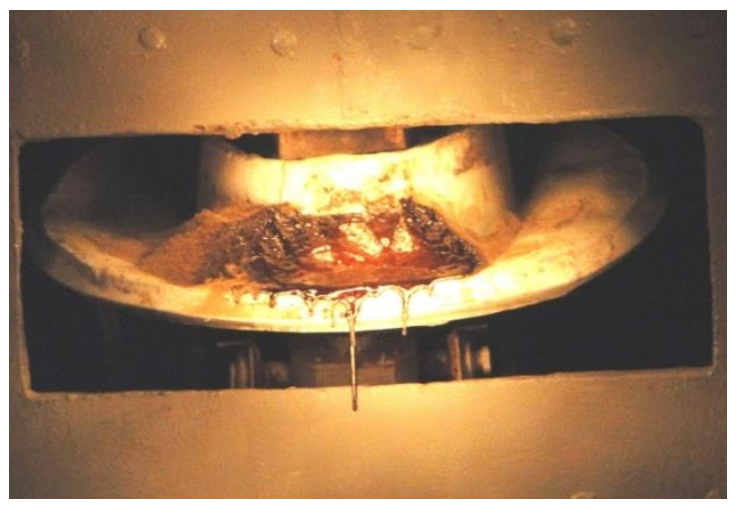

Fig. 2. The synthesis process of a refractory composite material in the focal zone of a BSF.

\section{Pyrometric method}

Pyrometric method for measuring the temperature of heated bodies. In practice, to measure high temperatures (up to $6000{ }^{\circ} \mathrm{C}$ and above), radiation pyrometers are used, the operation of which is based on the dependence of the spectrum and the total radiant energy of the 
radiation of heated bodies on temperature. A characteristic feature of radiation pyrometers is the ability to measure temperature at a distance, without the sensor contacting the measured medium. For this purpose, we use an innovative technology for measuring high temperatures of irradiated materials with a hightemperature radiant flux of solar radiation. This remote method was carried out on the basis of temperature measurement using an IG-12 TSP IR pyrometer manufactured by "IMPAC infrared GmbH" (Germany) with a laser designator [3]. The measurements are carried out from the laboratory room located in front of the focal plane, on the BSP concentrator at a distance of $20 \mathrm{~m}$. For further analysis of the experimental data, the density of the direct solar radiation flux is measured in parallel using an AT-50 actinometer [4].

A radiation pyrometer is a telescope consisting of a heat receiver and an optical system that concentrates on the heat receiver the total radiant flux emitted by the surface of the body whose temperature is to be measured. Usually, a thermopile is used as a heat receiver - several thermocouples connected in series, the working junctions of which are heated by the action of the radiant energy falling on them, and the free junctions take the temperature of the telescope body. The thermoelectric driving force of the battery is determined by the amount of heat $\mathrm{Q}$ received by its working junction, which, in accordance with the Stefan-Boltzmann law, is proportional to the fourth power of the absolute temperature $\mathrm{T}$ of the measured body [5]:

$$
Q=\frac{a_{s}}{\varepsilon} \cdot \sigma \cdot T^{4}
$$

where $a_{\mathrm{s}}$ - the relative coefficient of radiation absorption of the body, $\varepsilon$ - the relative coefficient of radiation of the body, the temperature of which is measured; $\sigma$ is the emissivity of an absolutely black body.

The principle of operation of pyrometric devices is based on the dependence of the emissivity of heated bodies or spectral intensity, on the absolute temperature. A pyrometer is a means or a set of temperature measuring instruments based on thermal electromagnetic radiation, designed to generate a signal of measuring information in a form convenient for direct perception by an observer. The design of pyrometers consists of a combination of a pyrometric transducer and a device for displaying information in analog or digital form.

Pyrometric methods for measuring the temperature of heated bodies have the following advantages [6]:

- the speed of measuring the temperature of heated bodies, which is determined by the type of radiation

receiver used and the circuit for processing electrical signals;

- the ability to measure the temperature of moving objects and equipment elements under high voltage;
- the absence of distortion of the temperature field of the controlled object, which is especially important when measuring the temperature of materials with low thermal conductivity such as ceramics, wood, plastic, as well as the risk of surface damage and shape for soft objects;

- the ability to measure high temperatures, at which the use of contact measuring instruments is either impossible or their operating time is very short;

- the ability to work in an aggressive environment, increased radiation and high ambient temperatures when the receiving head and the electronics of the pyrometer are separated using a fiber-optic cable.

The main disadvantage of pyrometric temperature measurements is the difficulty of taking full account of the relationships between the thermodynamic temperature of an object and the thermal radiation recorded by the pyrometer. It is necessary to take into account the change in the surface emissivity on the wavelength in the recorded spectral range and on the temperature in the measurement range, the presence of radiation absorption in the medium between the pyrometer and the object of control, the geometric parameters of the pyrometer's field of view and its optical system, the temperature of the environment and the device body.

\section{PYROMETER IG-12-TSP THAT WE OPERATE}

The IG-12-TSP pyrometer is used together with the INFRAWIN 5 program, which allows visualizing temperature changes in the form of a graph and a table. The same results can be saved in the computer memory using this interface.

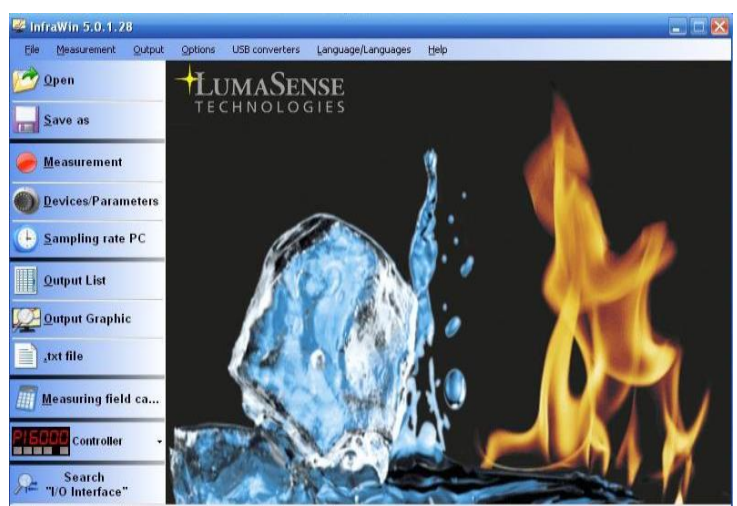

Figure: 3 . General view of the InfraWin 5 software. 


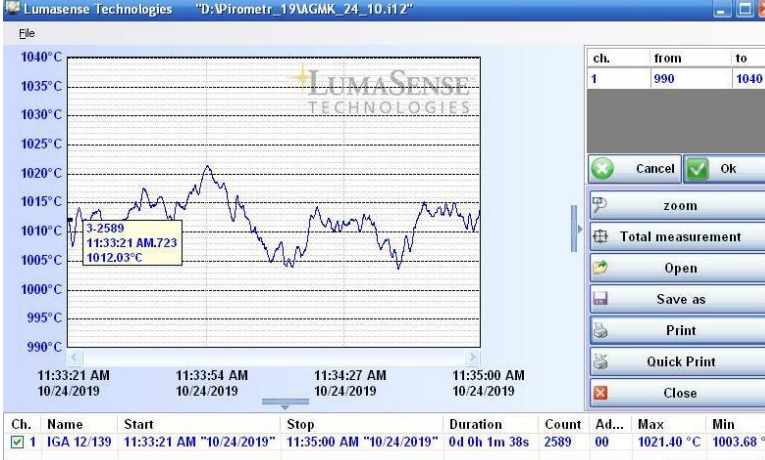

Fig. 4. The saved data in the form of a graph. Heat treatment of material under a concentrated flow at a temperature over $1000{ }^{\circ} \mathrm{C}$.

The program allows you to enter custom parameters: $\varepsilon^{-}$material emissivity, temperature measurement range, sampling rate for temperature measurement, etc. Sensitivity spectrum -1.57 microns. The maximum measurement temperature is up to 3000 degrees. Measurement accuracy up to $1500{ }^{\circ} \mathrm{C} 0.15 \%$ and higher than 1500 degrees $0.25 \%$.
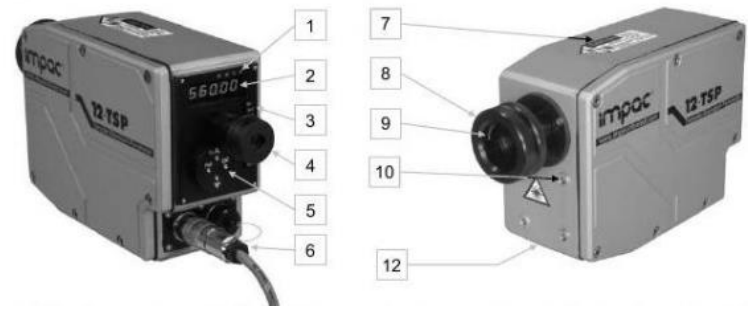

Fig. 5. General view of the IGA 12-TSP pyrometer. 1 - Display of temperature in $\mathrm{C}$ or $\mathrm{F}$ and active limit switches; 2 - LED display for showing measured temperature or parameters; 3 - parameter indicator; 4 viewfinder; 5 - setting button; 6 - Connecting cable; 7 Laser warning plate; 8 - Focusable optics; 9 - Optics information label; 10 - mounting holes for accessories; 12 - Label type (underside of the device).

Laser aiming light. For easy alignment with the measured object, the pyrometer is equipped with a laser sight - visible red light with a wavelength of 630 to 680 $\mathrm{nm}$ and a maximum power of $1 \mathrm{~mW}$. This pointer allows you to scan the irradiated or synthesized material up to $1.6 \mathrm{~m}$ in diameter with a resolution of $2 \mathrm{~mm}$.

Emissivity. For correct measurement, you need to adjust the emissivity. This emissivity is the ratio of the radiation of a real object and the radiation of a black body source at the same temperature. Different materials have different emissivities ranging from $0 \%$ to $100 \%$ (pyrometer settings from 10 to $100 \%$ ).
Emissivity depends on the surface condition of the material, the spectral range of the pyrometer and temperature meter. The emissivity of the pyrometer should be adjusted according to the typical value of the given material (see table 1).

Main characteristics of the IGA 12-TSP pyrometer. Areas and objects of measurement: Control of melting, synthesis and heat treatment of materials under a concentrated radiant stream of the sun; Temperature measurement range: $700-3000{ }^{\circ} \mathrm{C}$; Spectral measurement range: $1.45 \div 1.81$ microns; Optical Resolution: Distance to Image Size 900: 1; Temperature measurement error: $0.25 \%$.

The emissivity tolerance for each material is mainly dependent on surface conditions. Rough surfaces have a higher emissivity.Incorrectly entered emissivity of the heat-treated material into the pyrometer can lead to significant errors in temperature measurement. Therefore, we have created and are constantly updating a database of emissivity in various temperature ranges of often synthesized and heat-treated materials in the focal zone of BSFs (see Table 1).

Table 1. Emissivity of the most commonly synthesized materials in the focus of BSF.

\begin{tabular}{|l|l|l|}
\hline Material Name & Temperature, ${ }^{\circ} \mathrm{C}$ & $\begin{array}{l}\text { Coefficient } \\
\text { emissivity }\end{array}$ \\
\hline $\mathrm{Al}_{2} \mathrm{O}_{3}$ & 1227 & 0,40 \\
\hline $\mathrm{MgO}$ & 1227 & 0,31 \\
\hline $\mathrm{ZrO}_{2}$ & 1227 & 0,41 \\
\hline Copper oxide & $800-1100$ & $0,66-0,54$ \\
\hline Nickel oxide & $650-1255$ & $0,58-0,86$ \\
\hline Iron oxide & $500-1200$ & $0,85-0,95$ \\
\hline
\end{tabular}

\section{Conclusion}

Measuring the temperature of synthesized materials in the focus of the BSF using innovative measurement technologies using an IR pyrometer with a laser designator and a special program allows you to remotely measure with high accuracy, control and record in the computer memory the temperatures of heated bodies under a highly concentrated flow of solar radiation.

\section{References}

1. Nazarov, VI Heat engineering measurements and instruments: textbook. allowance / V.I. Nazarov. Minsk: Higher school, 2017 .- 280 p. : IL. ISBN 978985-06-2801-5. 
2. Rakhimov R.Kh. Big Solar Furnace. // Computational nanotechnology. Vol. 6, No. 2, 2019 S. 141-150. ISSN 2313-223X DOI: 10.33693 / 2313-223y-2019-6-2-141150.

3.https: //all-pribors.ru/opisanie/43275-09-is-12-tsp-iga12-tsp-45411

4. Fayziev Sh.A., Sobirov Yu.B. and other Measuring instruments for actinometric observations // Standart. 2017.No. 3, p. 45-47.

5. Quarton L.A. Stefan-Boltzmann radiation law. Study guide for laboratory work in the discipline "Physics workshop" / Far Eastern Federal University. Vladivostok: Far East. federal un-t, 2013 .-- 16 p. 6.https://studme.org/252438/tehnika/pirometry_izlucheniya 